\title{
Check It Out: On the Efficient Formal Verification of Live Sequence Charts`
}

\author{
Jochen Klose ${ }^{3}$, Tobe Toben ${ }^{1}$, Bernd Westphal ${ }^{1}$, and Hartmut Wittke ${ }^{2}$ \\ ${ }^{1}$ Fak. II, Dept. für Informatik, Carl von Ossietzky Universität Oldenburg, \\ 26111 Oldenburg, Germany \\ \{toben, westphal\}@informatik.uni-oldenburg.de \\ 2 OSC - ES AG, Industriestr. 11, 26121 Oldenburg, Germany \\ wittke@osc-es.de \\ ${ }^{3}$ Bombardier Transportation, Wolfenbüttler Str. 86/Obergstr. 5, \\ 38102 Braunschweig, Germany \\ jochen.klose@de.transport. bombardier.com
}

\begin{abstract}
Live Sequence Charts (LSCs) are an established visual formalism for requirements in formal, model-based development, in particular aiming at formal verification of the model. The model-checking problem for LSCs is principally long solved as each LSC has an equivalent LTL formula, but even for moderate sized LSCs the formulae grow prohibitively large. In this paper we elaborate on practically relevant subclasses of LSCs, namely bonded and time bounded, which don't require the full power of LTL model-checking. For bonded LSCs, a combination of observer automaton and fixed small liveness property and for additionally time bounded LSCs reachability checking is sufficient.
\end{abstract}

\section{Introduction}

Scenario-based approaches in general and Live Sequence Charts (LSCs) [1] in particular have shown adequate for the formal specification of inter-object requirements on distributed systems in formal, model-based development(cf. [2] for references). That is, requirements on a system under design are formally specified by LSCs before a model of the system is built. Model-checking can then automatically check whether the model satisfies the requirements to find errors early, before the actual implementation.

The model-checking problem of LSCs vs. Kripke structures is principally solved since universal LSCs translate to equivalent LTL formulae [3] and existential LSCs translate to observer automata, i.e. 1-acceptance 4, or CTL* thus corresponding model-checkers can directly be employed. Accordingly there are proof-of-concept results for the formal verification of LSCs against Statemate 56] and UML [78 models. In particular with the industrial case study considered in [5] it turned out that the LTL formulae grow large even for LSCs of moderate size. That is, formal verification becomes expensive due to the size

\footnotetext{
* Partly supported by DFG, grants SFB/TR 14 AVACS and DA 206/7-3, SPP 1064.
} 
of the requirements (for details cf. 39]). Our subject is efficient formal verification of LSCs. We identify two sub-classes of LSCs for which techniques that are faster but less powerful than LTL model-checking are sufficient, or help in finding errors fast.

Related Work. Model-checking LSCs against system models has been first investigated by [10]. They manually derive a selection of small, local LTL properties from an LSC and check whether they hold for a model of a bus protocol. The limited size of the checked properties didn't raise the need to consider more efficient procedures. In [11, model-checking is used as a technique to obtain satisfying paths for a set of LSCs in the context of playing-out LSCs [12]. Their representation of LSCs employs one automaton per instance-line. Similarly, [13, check a set of LSCs for consistency using a CSP semantics of LSCs, namely one CSP process per instance line, and the FDR model-checker. Both are particularly tailored for checking LSCs against each other and don't discuss the relation to general system model. Furthermore, both discuss only a limited subset of the dialect [5], in particular excluding time. We use the term "LSC verification" similar to, for instance, "LTL verification" which means checking a formula against a model. The observer based approach for LSC model-checking has been introduced in [14 and further studied in the context of Symbolic Timing Diagrams (STDs) in [15]. Our results slightly extend [15] since we have to discuss the case of non-deterministic automata which are needed for non-bonded LSCs while deterministic automata are sufficient for the scope of [15].

The remainder of the paper is structured as follows. In Sect. 2 we briefly recall LSCs. Section 3 introduces Timed Symbolic Automata (TSA), the semantical foundation of LSCs, together with the notions of determinism and time boundedness. It provides the basic strategy for efficient verification of TSAs. The application to LSC model-checking is discussed in Sect. 4 Section 5 supplies experimental results and Sect. 6] concludes.

\section{Live Sequence Charts}

The visual formalism LSCs has been introduced in 1 to overcome several shortcomings of the well-known Message Sequence Charts (MSC) wrt. a formal usage.

Table 1. Modalities of LSC elements and their semantics

\begin{tabular}{l|l|l|} 
& \multicolumn{1}{|c|}{ mandatory/hot/universal } & \multicolumn{1}{c|}{ possible/cold/existential } \\
\hline chart & $\begin{array}{l}\text {. . each activating system run } \\
\text { suffix adheres to scenario }\end{array}$ & $\begin{array}{l}\text {. there is an activating and ad- } \\
\text { hering system run suffix }\end{array}$ \\
\hline location & progress enforced & progress not enforced \\
\hline $\begin{array}{l}\text { condition/ } \\
\text { local invar. }\end{array}$ & $\begin{array}{l}\text { system violates LSC if condition } \\
\text { doesn't hold }\end{array}$ & $\begin{array}{l}\text { system satisfies LSC if condition } \\
\text { doesn't hold }\end{array}$ \\
\hline message & reception has to be observed & reception needn't be observed \\
\hline
\end{tabular}


It is a conservative extension of basic MSCs that gains increased expressive power by adding modalities to charts, locations, and elements (cf. Table 1).

The mode of a chart can be either existential or universal. An existential LSC is satisfied by a system if there is at least one system run adhering to the LSC. Conversely, a universal LSC is satisfied if all runs of the system adhere to it. A location's mode, either hot or cold, expresses liveness requirements. An element following a hot location has to be observed finally in order to satisfy the LSC. A cold location doesn't enforce progress. Conditions are, in contrast to MSCs, semantically relevant in LSCs and have a mode. If a mandatory (or hot) condition isn't satisfied when supposed to according to the scenario, the chart is violated. If a possible (or cold) condition isn't satisfied, the whole chart is immediately considered satisfied. It is legally exited. This interpretation applies alike to local invariants. They have been introduced in [5] to state requirements on spans of time instead of only single points in time as with conditions. The mode of a message, either mandatory (hot) or possible (cold), denotes whether the message may get lost. The reception of a hot message has to be observed to satisfy the LSC, for a cold message it needn't be observed.

In addition to modes, LSCs add to MSCs means that characterise the situations to which the scenario applies, i.e. its activation. Activation in general is characterised by a prefix of the LSC called pre-chart meaning whenever the pre-chart is observed then the system shall adhere to the rest of the LSC, the main-chart. The activation condition is a shortcut for pre-charts with a single condition only. For formal verification, [5] adds the activation mode - one of initial, invariant, or iterative - to further restrict activation. Initial LSCs may only be activated in initial system states. Iterative LSCs disregard violations of reactivating LSCs, i.e. LSCs that comprise a sequence that adheres to the LSC's own pre-chart again. Furthermore, both 512 have added the notion of strict vs. tolerant (or weak) interpretation. The strict interpretation requires that messages used in the chart don't occur at other points in time than the ones given by the LSC. The tolerant interpretation ignores additional messages. For example, a system sending red_on once again before expiration of the timer would not satisfy the LSC from Fig. 1 strictly.

Fig. 1(a) is a simplified requirement on a level crossing controller. It is activated when the crossing controller receives an asynchronous message 'secreq' from the environment. The crossing controller shall finally, as indicated by the solid segment of its instance line, start the lights and barrier controllers by synchronous messages 'lights_on' and 'barrier_down'. The timing interval $[5,15]$ requires lowering the barrier to take between 5 and 15 units of time and the hot local invariant $\neg M v U p$ requires the barrier not to move up from 'barrier_down' reception up to and including the point in time where 'barrier_ok' is sent. If the traffic lights controller is not operational when receiving 'lights_on', the LSC is legally exited at the cold condition 'Operational'. Otherwise timer $t$ is started. Timeout of $t$ shall occur when sending 'lights_ok', i.e. switching on the lights shall take exactly 7 units of time. The order of 'lights_ok' and 'barrier_ok' is explicitly relaxed by enclosing them in a coregion as indicated by the dotted line. 


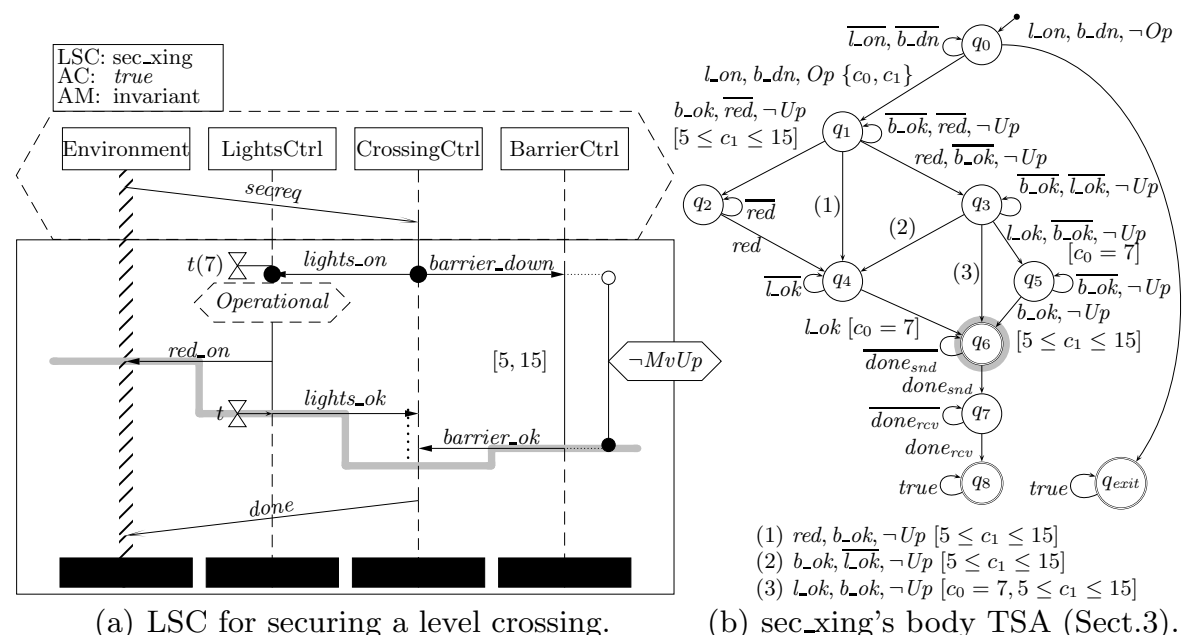

Fig. 1. For brevity, overlining denotes negation and comma denotes conjunction in Fig. 1(b) E.g. $q_{0}$ 's loop fires if neither 'lights_on' nor 'barrier_down' are observed.

The messages may occur in any order, even simultaneously. When both have been received, the crossing controller may send 'done' back to the environment as no hot location enforces progress at this position.

We postpone recalling the formal semantics of LSC following [5] to Sect. 4] thus after the introduction of Timed Symbolic Automata in Sect. 3, Note that we discuss the LSC dialect of [5] which is tailored for the application domain of formal verification in contrast to the play-engine dialect of [12]. This is not an exclusive choice as both share a large common sublanguage. LSC specifications may well be played in following [12] and then strengthened for formal verification using the features from [5]. Furthermore we assume well-formed LSCs [2], i.e. LSCs without internal contradictions.

\section{Efficient TSA Model-Checking}

\subsection{Preliminaries}

We use $\operatorname{Expr}_{\mathcal{S}}$ to denote the propositional logic formulae over signature $\mathcal{S}$, and $\mathcal{I} \models \psi$ to denote that interpretation $\mathcal{I}$ satisfies $\psi \in$ Expr $_{\mathcal{S}}$ with fixed nonempty universe $\mathcal{U}$. Let $C$ be a set of clocks. A clock valuation is a mapping $\tau: C \rightarrow \mathbb{N}_{0}$, and $T(C)$ denotes the set of all clock valuations. The update of a time valuation $\tau \in T(C)$ by a value $x \in \mathbb{N}_{0}$, written $\tau+x$, is pointwise defined as $(\tau+x)(c):=\tau(c)+x$ for all $c \in C$. The set of clock constraints $\Phi(C)$ is defined by the grammar $\phi::=$ true $|c \leq x| c \geq x, \varphi::=\phi\left|\varphi_{1} \wedge \varphi_{2}\right| \varphi_{1} \vee \varphi_{2}, c \in C$, $x \in \mathbb{N}_{0}$. We write $\tau=\varphi$ to denote that the clock valuation $\tau \in T(C)$ satisfies the clock constraint $\varphi \in \Phi(C)$. The definition of satisfaction is standard. 


\subsection{Timed Symbolic Automata}

Timed Symbolic Automata (TSA) are a variant of timed Büchi automata where transitions are labelled by expressions from Expr $r_{\mathcal{S}}$ instead of just an element of an alphabet (cf. 15] for references). The new notion of default transitions significantly eases the formal definition of the clock propagation introduced below.

Formally, a TSA over a signature $\mathcal{S}$ is a tuple $\mathcal{A}=\left(Q, q_{s}, C, \rightsquigarrow, D, F\right)$ with a finite set of states $Q$, initial state $q_{s} \in Q$, a finite set of clocks $C$, transition relation $\rightsquigarrow \subseteq Q \times \operatorname{Expr}_{\mathcal{S}} \times \Phi(C) \times 2^{C} \times Q$, default transitions $D \subseteq Q \times Q$, and accepting states $F \subseteq Q$. We define $\rightarrow \subseteq Q \times Q$ as $\rightarrow:=D \cup\left\{\left(q, q^{\prime}\right) \mid\right.$ $\left.\exists\left(q, \psi, \varphi, \rho, q^{\prime}\right) \in \rightsquigarrow\right\}$. A TSA is called Partially Ordered TSA (POTSA) if the reflexive transitive closure of $\rightarrow$ is a partial order, i.e. $\rightarrow^{*}$ is anti-symmetric. Note that all loops in a POTSA are consequently self-loops.

Let $\mathcal{S}$ be a signature and $\mathcal{U}$ a fixed universe. A timed interpretation sequence is a sequence $r=\left(\left(\iota_{i}, t_{i}\right)\right)_{i \in \mathbb{N}_{0}}$ with $\iota_{i}$ an interpretation of $\mathcal{S}$ and $t_{i} \in \mathbb{N}_{0}$ a timestamp such that $t_{i}<t_{i+1}, i \in \mathbb{N}_{0}$. Let $\left(\left(q_{i}, \tau_{i}\right)\right)_{i \in \mathbb{N}_{0}}$ be a sequence with $q_{i} \in Q$ a state and $\tau_{i} \in T(C)$ a valuation of the clocks, $i \in \mathbb{N}_{0}$. It is called timed run of $\mathcal{A}$ over $r$ iff it starts in the initial state, i.e. $q_{0}=q_{s}$, the clocks initially have value zero, i.e. $\tau_{0}(c)=0, c \in C$, and states are $\mathcal{A}$-successors. That is, for $i \in \mathbb{N}_{0}$ either there is a transition $\left(q_{i}, \psi_{i}, \varphi_{i}, \rho_{i}, q_{i+1}\right) \in \rightsquigarrow$ such that the boolean and clock constraints hold, $\iota_{i} \models \psi_{i}$ and $\left(\tau_{i}+\left(t_{i+1}-t_{i}\right)\right) \models$ $\varphi_{i}$, and the clock valuations are updated according to $\rho_{i}$, i.e. $\left.\tau_{i+1}\right|_{\rho_{i}}=0$ and $\left.\tau_{i+1}\right|_{C \backslash \rho_{i}}=\left.\left(\tau_{i}+\left(t_{i+1}-t_{i}\right)\right)\right|_{C \backslash \rho_{i}}$, or there is a default transition $\left(q_{i}, q_{i+1}\right) \in D$ and $\tau_{i+1}=\tau_{i}+\left(t_{i+1}-t_{i}\right)$. A timed run $\left(\left(q_{i}, \tau_{i}\right)\right)_{i \in \mathbb{N}_{0}}$ is called accepting if $q_{i} \in F$ for infinitely many $i \in \mathbb{N}_{0}$. The language accepted by $\mathcal{A}$, denoted by $\mathcal{L}(\mathcal{A})$, is the set of timed interpretation sequences for which an accepting run exists.

In the following we introduce two subclasses of TSAs, namely deterministic and time-bounded ones. In Sect. 3.4 we will see how membership in these classes determines the efficiency of the model-checking procedure.

We call a state $q \in Q$ determinstic if the constraints on all outgoing transitions are mutually disjoint, i.e. for each two transitions $\left(q, \psi_{1}, \varphi_{1}, \rho_{1}, q_{1}\right),\left(q, \psi_{2}\right.$, $\left.\varphi_{2}, \rho_{2}, q_{2}\right) \in \rightsquigarrow$ with $q_{1} \neq q_{2}$ we have $\left(\iota \models \psi_{1} \wedge \tau \models \varphi_{1}\right) \rightarrow \neg\left(\iota \models \psi_{2} \wedge \tau \mid=\varphi_{2}\right)$ for any $\iota$ and $\tau$. It is called reaching-deterministic if all $q^{\prime} \in Q$ with $q^{\prime} \rightarrow^{*} q$ are deterministic. We call $\mathcal{A}$ determinstic iff all its states are deterministic.

Given a set of clocks $C$, the set of upper bounded clock constraints $\Phi_{\triangleleft}(C) \subseteq$ $\Phi(C)$ is defined by the grammar $\phi::=x_{1} \leq c \wedge c \leq x_{2}, \varphi::=\phi\left|\varphi_{1} \wedge \varphi_{2}\right| \varphi_{1} \vee \varphi_{2}$, $c \in C, x_{1}, x_{2} \in \mathbb{N}_{0}$. We call a state $q \in Q$ time bounded iff the clock constraints on all outgoing transitions are from $\Phi_{\triangleleft}(C)$. We call $\mathcal{A}$ time bounded iff all states from $Q \backslash F$ are time bounded. Now let $\mathcal{A}$ be a POTSA and $q$ a state of it s.t. all outgoing transitions impose a finite upper bound on clock $c$. Let $q^{\prime}$ be a state from which an accepting state is only reached by visiting $q$ and let $c$ not be reset along the path from $q^{\prime}$ to $q$. Then the boundedness of $c$ transitively induces bounds on all transitions between $q^{\prime}$ and $q$, including self-loops [15]. For example, clock constraint $c_{0}=7$ at the transition from state $q_{4}$ to $q_{6}$ in Fig. 1(b) propagates to the transition from $q_{2}$ to $q_{4}$ and to the self-loops at $q_{4}$ and $q_{2}$. Consequently, the number of time bounded states can be increased by 
propagating clock constraints backwards through the automaton, i.e. making the implicit constraints explicit. In [15] we give an algorithm which performs clock propagation on POTSAs and yields a language-equivalent POTSA.

Another operation of interest is the failure state completion [15] of $\mathcal{A}$. For each reaching-deterministic state in $Q$, it adds a default transition to a designated failure state ' $q_{\text {fail }}$ ', i.e. it yields the language equivalent [15] TSA $\left(Q \dot{\cup}\left\{q_{f a i l}\right\}, q_{s}, C\right.$, $\left.\rightsquigarrow, D^{\prime}, F\right)$ with $D^{\prime}:=D \cup\left\{\left(q, q_{\text {fail }}\right) \mid q\right.$ is reaching-deterministic $\}$. Then reaching ' $q_{\text {fail }}$ ' with sequence $r$ is a sufficient criterion for $r \notin \mathcal{L}(\mathcal{A})$.

\subsection{Timed Symbolic Automata as Specification}

A Kripke Structure is a tuple $K=\left(A P, S, S_{0}, R, L\right)$ with atomic propositions $A P$, states $S$, initial states $S_{0} \subseteq S$, transition relation $R \subseteq S \times S$, and labelling function $L: S \rightarrow 2^{A P}, A P$ and $S$ finite. As TSAs are defined using interpretation sequences, we assume that each subset $\ell \subseteq A P$ defines an interpretation $\iota_{\ell}$ of $\mathcal{S}$. The TSA and the model are then called compatible. A run $s_{0}, s_{1}, \ldots$ of $K$ induces the timed interpretation sequence $\left(\left(\iota_{i}, t_{i}\right)\right)_{i \in \mathbb{N}_{0}}$ with $\iota_{i}=L\left(s_{i}\right), t_{i}=i$. The set of all timed interpretation sequences induced by the runs of $K$ is denoted by $R(K)$.

Using a TSA $\mathcal{A}$ as a specification on $K$ means relating $R(K)$ to $\mathcal{L}(\mathcal{A})$. To increase precision of the specification, here we always consider an activation condition $\nu \in \operatorname{Expr}_{\mathcal{S}}$ and an activation mode of initial, invariant, and iterative together with $\mathcal{A}$. We say $K$ satisfies $\mathcal{A}$ initially wrt. $\nu$, denoted by $\left.K\right|_{\nu \text {,init }} \mathcal{A}$, iff all $\left(\left(\iota_{i}, t_{i}\right)\right)_{i \in \mathbb{N}_{0}} \in R(K)$ with $\iota_{0} \models \nu$ are in $\mathcal{L}(\mathcal{A})$. It satisfies $\mathcal{A}$ invariantly wrt. $\nu$, denoted by $K \models_{\nu, \text { inv }} \mathcal{A}$, iff $\iota_{i}=\nu$ implies that the suffix $\left(\vec{\iota}_{i+1}, \vec{t}_{i+1}\right)\left(\vec{\iota}_{i+n}, \vec{t}_{i+n}\right) \ldots$ is in $\mathcal{L}(\mathcal{A})$. Iterative satisfaction, denoted by $K \models_{\nu, i t e r} \mathcal{A}$, is special to POTSAs. It is similar to invariant but excludes overlapping activations of $\mathcal{A}$. The motivation to introduce this mode was to ease the understanding of counter-examples. It is easier to uniquely identify where activation takes place if there are no overlapping activations. But this mode has the serious drawback that if it is used for a TSA that actually has an overlapping activation, a violation may be shadowed. Checking whether a TSA doesn't have an overlapping activation (it is then called non-reactivating) or not is an additional non-trivial task.

Note that by the definition above we choose model steps as time units. In general, other notions of time have to be supported, for instance, the supersteps of Statemate models. In [15], the approach presented here has been extended to support two notions of time by assuming that the passing of time is observable on the model, for instance by a special signal of the model which is raised whenever a superstep is completed. To keep the discussion focused and to adhere to space restrictions, we only consider step time. Adding the more general treatment of time following [15] is actually straightforward.

\subsection{Efficient POTSA Model-Checking}

Each non-iterative POTSA with step clocks has an equivalent LTL formula [16] in negative normal form, thus LTL model-checking can be applied to decide whether $K$ satisfies $\mathcal{A}$. For all POTSAs, there is an additional approach based on composing in parallel to the model a number of time counters, one for each 


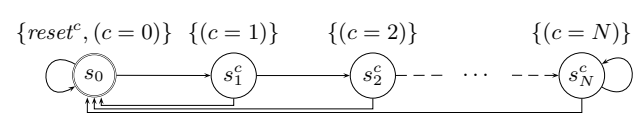

Fig. 2. Kripke structure of timer $c$ with upper bound $N+1$

clock, and a kind of transition system view of the TSA, i.e. dismissing the Büchi criterion. This parallel composition is then checked for reachability of certain states or for a small fixed liveness formula. In this section we introduce the parallel composition of $K$ with observers. Section 3.4 .3 discusses when to apply which approach.

\subsubsection{Timer Extension}

In model-checking timed TSA properties, we can't directly employ the sequences from $R(K)$ as the time stamps are unbounded. By [15] it is sufficient for the observer approach to consider finite time counters for each clock of the (not necessarily time bounded) TSA and these bounds are effectively computable. These time counters are transition systems that count model steps until their finite upper bound is reached and that may reset themselves any time and then set a reset flag (cf. Fig. 2). They are composed in parallel to the model. More formally, let $c$ be a clock with upper bound $N+1$, and $S_{c}=\left\{s_{0}^{c}, \ldots, s_{N}^{c}\right\}$ a set of fresh states wrt. $S$. The timer extension of $K$ for $c$ is $K^{c}=\left(A P^{c}, S^{c}, S_{0}^{c}, R^{c}, L^{c}\right)$ with

$$
\begin{gathered}
-A P^{c}=A P \cup\left\{\text { reset }^{c},(c=0), \ldots,(c=N)\right\}, S^{c}=S \times S_{c}, S_{0}^{c}=S_{0} \times\left\{s_{0}^{c}\right\}, \\
-R^{c}=\left\{\left(\left(s, s_{i}^{c}\right),\left(s^{\prime}, s_{0}^{c}\right)\right),\left(\left(s, s_{N}^{c}\right),\left(s^{\prime}, s_{N}^{c}\right)\right) \mid\left(s, s^{\prime}\right) \in R\right\} \\
\cup\left\{\left(\left(s, s_{i}^{c}\right),\left(s^{\prime}, s_{i}^{c}+1\right)\right) \mid\left(s, s^{\prime}\right) \in R, 0 \leq i<N\right\} \\
-L^{c}\left(\left(s, s_{i}^{c}\right)\right)=L(s) \cup\left\{\text { reset }^{c} \mid i=0\right\} \cup\{(c=i)\} .
\end{gathered}
$$

The timer extension $K^{c}$ is by the same procedure extended to $K^{c, c^{\prime}}$ for a second clock. We use $K^{C}:=K^{c_{1}, \ldots, c_{n}}$ to denote the timer extension of $K$ for all clocks from $C$. A state $s \in K^{C}$ canonically defines a clock valuation $\tau_{s}$ as $\tau_{s}(c):=i$ if $(c=i) \in L(s)$. The clocks reset in $s$ are $\rho_{s}:=\left\{c \mid\right.$ reset $\left.^{c} \in L(s)\right\}$.

\subsubsection{Observer Extension}

Let $\mathcal{A}=\left(Q, q_{s}, C, \rightsquigarrow, D, F\right)$ be a TSA compatible with $K$ and $K^{C}$ the timer extension of $K$. Using $s_{C}$ to denote the components of states that are introduced by the timer extension, the observer extension of $K$ for activation expression $\nu$ and initial activation $\mathcal{A}$ is $K^{\mathcal{A}} /$ init $=\left(A P^{\mathcal{A}}, S^{\mathcal{A}}, S_{0}^{\mathcal{A}}, R^{\mathcal{A}}, L^{\mathcal{A}}\right)$ with

- $A P^{\mathcal{A}}=\left(A P^{C} \dot{\cup}\{\right.$ fair, fail $\left.\}\right), S^{\mathcal{A}}=S^{C} \times\left(Q \dot{\cup}\left\{q_{\text {idle }}\right\}\right)$,

$-S_{0}^{\mathcal{A}}=\left\{\left(s, s_{C}, q_{s}\right) \mid\left(s, s_{C}\right) \in S_{0}^{C}, \iota_{L(s)} \models \nu\right\}$ $\cup\left\{\left(s, s_{C}, q_{\text {idle }}\right) \mid\left(s, s_{C}\right) \in S_{0}^{C}, \iota_{L(s)} \not \models \nu\right\}$,

$-\left(\left(s, s_{C}, q\right),\left(s^{\prime}, s_{C}^{\prime}, q^{\prime}\right)\right) \in R^{\mathcal{A}}$ iff $\left(\left(s, s_{C}\right),\left(s^{\prime}, s_{C}^{\prime}\right)\right) \in R^{C}$ and $q=q^{\prime}=q_{\text {idle }}$ or

- either there is a regular transition $\left(q, \psi, \varphi, \rho, q^{\prime}\right) \in \rightsquigarrow$ with $\iota_{L(s)} \models \psi, \tau\left(s^{\prime}, s_{C}^{\prime}\right) \models \varphi$, and $\rho_{s}=\rho$

- or there is a default transition $\left(q, q^{\prime}\right) \in D$ and $\rho_{s}=\emptyset$,

$-L^{\mathcal{A}}\left(\left(s, s_{C}, q\right)\right)=L^{C}\left(\left(s, s_{C}\right)\right) \cup\{$ fair $\mid q \in F\} \cup\left\{\right.$ fail $\left.\mid q=q_{\text {fail }}\right\}$. 
For invariant activation, the observer $K^{\mathcal{A}} /$ inv is activated non-deterministically thus additionally all $\left(s, s_{C}, q_{i d l e}\right)$ are in $S_{0}^{\mathcal{A}}$ independent from satisfaction of $\nu$. Furthermore there is a transition from $q=q_{\text {idle }}$ to $q^{\prime}=q_{s}$ whenever $\iota_{L(s)} \models \nu$. For iterative activation, the initial states of the observer extension are as in $K^{\mathcal{A}} /$ init. $K^{\mathcal{A}} /$ iter remains in $q_{\text {idle }}$ only if $\nu$ is not satisfied and takes the transition to $q_{s}$ whenever possible. In addition there are transitions back to $q_{\text {idle }}$ and $q_{s}$ from each accepting state with only a self-loop. The transition is to $q_{s}$ if $\nu$ is satisfied and to $q_{\text {idle }}$ otherwise. Thus $K^{\mathcal{A}} /$ iter is slightly smaller than $K^{\mathcal{A}} /$ inv . Note that $K^{\mathcal{A}} / m, m$ a mode, restricts the behaviour of the clocks, but not the behaviour of the model. In $K^{\mathcal{A}} / m$, clocks are only reset if there is a transition in the TSA with a corresponding reset set. $K^{\mathcal{A}} / m$ sets the flag 'fail' iff the failure state of the TSA is reached and 'fair' iff the TSA is in an accepting state. We call a state $s^{\mathcal{A}} \in S^{\mathcal{A}}$ a failure state iff fail $\in L^{\mathcal{A}}\left(s^{\mathcal{A}}\right)$ and fair state iff fair $\in L^{\mathcal{A}}\left(s^{\mathcal{A}}\right)$.

\subsubsection{Putting It All Together}

Now we can devise a strategy for deciding whether a given Kripke structure satisfies a failure state completed POTSA using four different standard modelchecking procedures of different worst-case complexity, namely reachability checking with safety observer, ACTL model-checking with and without observer, and LTL model-checking. The following Lemma states that the less powerful techniques are helpful for finding violations early and that they are sufficient for deterministic (time bounded) POTSAs.

Lemma 1. Let $\mathcal{A}$ be a POTSA, K a Kripke structure, $\nu$ a condition, $m$ a mode.

1. 15. If a failure state of $K^{\mathcal{A}} / m$ is reachable, then $K \forall_{\nu, m} \mathcal{A}$. If $\mathcal{A}$ is deterministic and time bounded then $K \forall_{\nu, m} \mathcal{A}$ implies reachability of a failure state.

2. [15] If $K^{\mathcal{A}} / m \models \mathrm{AGAF}$ fair, then $K=_{\nu, m} \mathcal{A}$. If $\mathcal{A}$ is deterministic then $K \models{ }_{\nu, m} \mathcal{A}$ implies $K^{\mathcal{A}} / m \models$ AG AF fair.

3. If $\mathcal{A}$ is non-deterministic, then there is no LTL formula $\lambda$ using only atomic proposition 'fair' s.t. $K^{\mathcal{A}} / m \not \forall_{\nu, m} \lambda$ implies $K \forall_{\nu, m} \mathcal{A}$.

Proof. (13) Assuming such a formula $\lambda$, exploit non-determinism in observer to construct a Kripke structure $K$ s.t. $K^{\mathcal{A}} / m$ doesn't satisfy $\lambda$ but $K \models{ }_{\nu, m} \mathcal{A}$.

Note that Lemma 113 implies that there is no known procedure to decide satisfaction of iterative non-deterministic TSAs because within an LTL formula we cannot, as with observer extensions, refer to the own state of activation.

Using that the ACTL formula obtained from an LTL formula in negative normal form by prefixing all modal operators by ' $A$ ' implies the LTL formula, we devise the strategy depicted in the following control flow diagram for the verification of POTSA specifications. The idea is to apply the procedure with the best worst-case complexity first to find contradictions early. Only if no errors are unveiled and the procedure is too weak for the POTSA, the next expensive procedure is applied. The increasing prevalence of multi-processor or multi-core 
hosts allows to apply the procedures in parallel and stop all other procedures once a significant result is obtained.

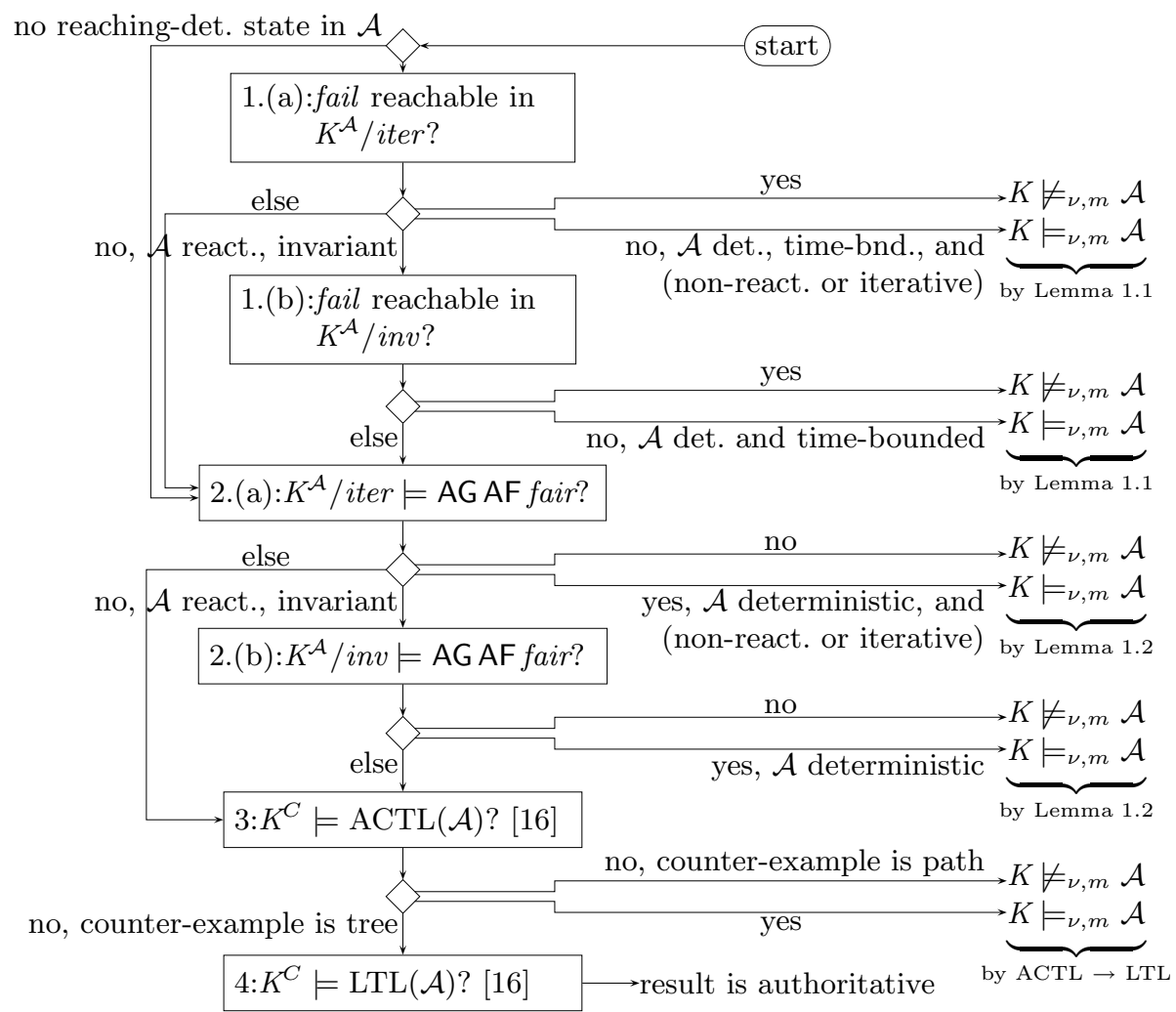

The refinement of steps 1 and 2 into (a) and (b) is a minor optimisation using the expectation that checking $\mathcal{A}$ iteratively is less expensive (cf. 3.4.2). An initial TSA is treated like an interative one, as the time of activation is then uniquely determined to be an initial state.

Note that the time and space resource consumption of certain observer based tasks can be reduced by applying a POTSA version of the decomposition algorithm presented in 9 and conducting the decomposed, smaller tasks in parallel.

\section{Efficient LSC Model-Checking}

The following transfer of the results from the previous section to the domain of LSCs is effective as most practically used LSCs yield deterministic POTSAs. Furthermore, unbounded liveness requirements typically only occur in early and rather abstract parts of a system's LSC specification and are restricted by bounds in later, more detailed versions thus most LSCs yield time-bounded TSAs.

Section 4.1 briefly recalls the LSC semantics in terms of TSA and relates the TSA classes identified in Sect. 3 to LSCs. For a complete definition of the LSC 


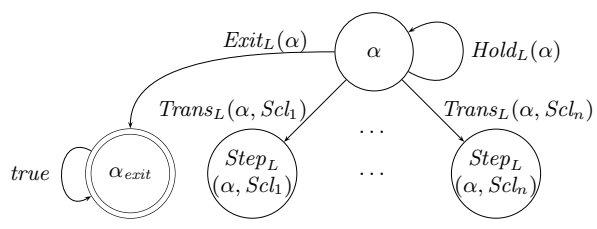

(a) Outgoing transitions from state $\alpha$.

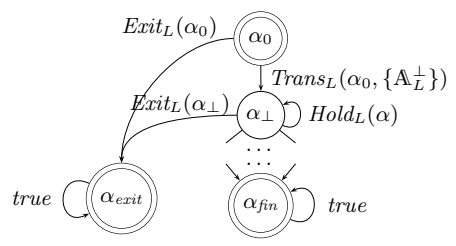

(b) Overall structure of $\mathcal{A}_{L}$.

Fig. 3. Structure of the LSC body automaton. Double lined states are in $F$.

language, its abstract syntax, and the semantics-giving unwinding procedure the reader is referred to [5]. The discussion of LSC verification starts in Sect. 4.2 with the simplest case, namely invariant universal LSCs without pre-chart or assumptions. The additional features of LSCs are then discussed in isolation in the subsequent sections.

\subsection{LSC Semantics and TSA Properties}

The central concept of the LSC semantics [5] is the notion of the cut, i.e. a set of at least one location per instance line (more than one for coregions). The gray line in Fig. 1(a) indicates a cut. Each TSA state corresponds to one cut of the LSC, e.g. the example cut in Fig. 1(a) corresponds to state $q_{6}$ in Fig. 1(b). Intuitively, a system satisfies the LSC if for each system run suffix with a prefix in the language of the pre-chart TSA, the rest of the run is in the language of the main-chart TSA. The algorithm of [5] translates the pre- and main-chart of each commitment and assumption LSC of a requirement into separate TSAs. Fig. 3(a) schematically shows a state of the TSA obtained by the unwinding procedure [5]. It has a self-loop awaiting the subsequent LSC elements and one transition for each combination of occurrence and non-occurrence of awaited elements. Violations of cold conditions lead to the accepting state $\alpha_{\text {exit }}$. Fig. $3(\mathrm{~b})$ shows the overall structure of the TSA. The state $\alpha_{\perp}$ corresponds to the cut with all instance heads and $\alpha_{\text {fin }}$ to complete traversal. Fig. 1(b) gives the TSA of the main-chart from Fig. 1(a), (The initial TSA state $\alpha_{0}$ is omitted for brevity.)

Following [3], each TSA of an LSC is a POTSA. The timer propagation algorithm of [15] applies directly. In [3], we have introduced a sufficient criterion on LSCs that implies determinism of the corresponding TSAs. The TSAs are deterministic if all conditions and local invariants occur bonded in the LSCs. That is, if they are in a simultaneous region with at least one message, timeout, or instance head. Then the evaluation time for the condition is well-defined. This criterion is easily [2] checkable on the abstract syntax of the LSC. The observer automaton construction of [15] as introduced in Sect. 3] applies directly as LSCs share activation modes initial, invariant, and iterative with TSAs.

\subsection{LSC Model-Checking}

Note that an LSC's strict or tolerant interpretation is mostly orthogonal to the issues discussed below. In the TSA, strictness is expressed by strenghtening the 
transition annotations. Each expression is additionally conjoined with a term that excludes all messages that are not referred to by the expression. Thus the strict TSA doesn't introduce new non-determinism and as it only considers messages, non-determism is not resolved except for few pathological cases. Consequently we needn't treat the interpretation explicitly below.

\subsubsection{Universal, No Pre-chart or Assumption}

In the easiest case, we only have to consider the TSA $\mathcal{A}$ of the main-chart and an activation condition. Then the algorithm from Sect. 3.4.3 applies directly.

\subsubsection{Pre-chart}

For the pre-chart of an LSC, a separate TSA is constructed. The slightly different algorithm adjusts transitions to the special interpretation of pre-charts which don't have a notion of violation. Using the ACTL/LTL way there are two options for checking an LSC with pre-chart. Let $\varphi_{p c}$ and $\varphi_{L}$ be the LTL formulae corresponding to the pre-chart and the whole LSC, i.e. pre- and main-chart together. Following [17, we can check $\mathrm{G}\left(\varphi_{p c} \rightarrow \varphi_{L}\right)$. This formula tends to grow large since the pre-chart part occurs twice, even if the more compact formulae also presented in [17] is usable.

As the semantics of the pre-chart is indication of complete traversal of its scenario, the Büchi criterion is actually not needed. Finite automaton acceptance is sufficient. This can be exploited by composing in parallel with the observer extension of a model a (non-deterministically activating) observer for the prechart that drives an additional proposition ' $p c$ ' which holds iff the pre-chart has just been observed. By changing the main-chart observer such that its activation expression is ' $p c$ ' the algorithm from Sect. 3.4.3 still applies directly.

If the observer way is too weak, then $\mathrm{G}\left(p c \rightarrow \varphi_{m c}\right)$ (or the ACTL correspondence) is checked in steps 3 and $4 ; \varphi_{m c}$ being the main-chart's LTL formula.

\subsubsection{Assumptions}

The semantics of LSC assumptions is standard. A system satisfies a universal LSC with assumptions iff all runs satisfying all assumptions also satisfy the commitment. Thus, iff

$$
\left(\mathrm{G}\left(\varphi_{a_{1}} \wedge \cdots \wedge \varphi_{a_{m}}\right)\right) \rightarrow\left(\mathrm{G}\left(p c \rightarrow \varphi_{m c}\right)\right)
$$

with $\varphi_{a_{1}}, \ldots, \varphi_{a_{m}}$ the LTL formulae for the assumptions, ' $p c$ ' the pre-chart observer from Sect. 4.2.2, and $\varphi_{m c}$ the main-chart's LTL formula.

To avoid representing the assumption part as (again large) formulae, we can start with properties which are stronger than (1), i.e. that imply (1), but are easier to check. On the downside, obtaining a counter-example is then no longer directly significant as the counter-example may be a false negative. It has to be checked not to be spurious, for example by simulation.

An approach stronger than (11) is to consider the tableau of the parallel composition of all assumptions as an additional observer, i.e. parallel composed to the observer extension. The formula to check is then (AG AF afair) $\rightarrow$ (AG AF fair) (2) 
where 'afair' holds iff the tableau is in an accepting state. If (2) passes and if the LSC and all assumptions are bonded, then the system satisfies the LSC. A failure may be a false negative.

An approach stronger than (2) is to check for reachability of a state with $\neg$ afail $\wedge$ fail in the parallel composition of $K^{\mathcal{A}}$ with one iteratively activated observer automaton per assumption, i.e. not the tableaux. If such a state isn't reachable and if the LSC and all assumptions are bonded and time-bounded and no assumption is invariant, then the system satisfies the LSC.

With these three (non exhaustive) options, the algorithm from Sect. 4.2.1 can be extended as follows. Pragmatically we don't consider all of the possible combinations with procedures for the commitment. In step 1, only the first option is used for assumptions in order to obtain a reachability property. In each substep of 2 , the first option for assumptions can be tried first, followed by the second option from above. In steps 3 and 4, all three options can be tried subsequently, trading size of the formula for size of the model.

Note that a non-bonded assumption is typically easily violated by "clever choice" of transitions in the TSA. Therefore non-bonded assumptions in general are of limited use as an easily violated assumption excludes too many system runs from consideration; the requirement may even be trivially satisfied if all system runs are excluded. As a consequence, all LSCs used in assumption/guarantee style verification should be bonded.

\subsubsection{Existential LSCs}

Recall from Sect. 2 that an existential LSC is satisfied by a system iff there is at least one system run that adheres to the LSC. Using CTL*, we obtain an equivalent formula by prefixing the LTL formula with the existential quantifiers 'E' or 'EF' 3.

But the existential mode is different from the universal mode in that one wants to obtain a witness if the formula is satisfied. That is, similar to precharts, the Büchi criterion isn't used and thus the ACTL/LTL way is practically not relevant. Furthermore, in practice one is typically interested in an example system run that traverses the LSC completely [18] instead of taking a legal exit on a cold condition. To achieve all this, the states of the main-chart TSA are turned into non-accepting states except for the state corresponding to the final cut. If the LSC has a pre-chart, the main-chart TSA is again activated by the pre-chart observer ' $p c$ ' as discussed in Sect. 4.2.2. Verifying the existential LSC is then equivalent to reachability of the remaining unique accepting state under all given assumptions.

\section{Figures, Please}

Table 2 supports our claims with exemplary experimental results. It lists pure model-checking time, i.e. without constructing and loading the transition relation BDD, and without counter-example post processing, for all techniques discussed in Sect. 4(VIS 2.0 [Brayton et al.], Intel Xeon 3.06GHz, 4GByte). The 
Table 2. Experimental Results. Model-checking time in seconds.

\begin{tabular}{|c|c|c|c|c|c|c|}
\hline & rch/iter & rch/inv & AGAF/iter & AGAF/inv & ACTL & LTL \\
\hline Fig 1/tb & $36.3 \mathrm{~s} / \boldsymbol{X}^{*}$ & $48.6 \mathrm{~s} / \boldsymbol{X}$ & $303.4 \mathrm{~s} / \boldsymbol{X}$ & $294.9 \mathrm{~s} / \boldsymbol{X}$ & $755.2 \mathrm{~s} / \boldsymbol{X}$ & - \\
\hline Fig $1 /$ tb & $37.3 \mathrm{~s} / \boldsymbol{V}^{*}$ & $45.0 \mathrm{~s} / \mathrm{V}$ & $41.0 \mathrm{~s} / \boldsymbol{V}$ & $31.5 \mathrm{~s} / \boldsymbol{V}$ & $698.8 \mathrm{~s} / \boldsymbol{V}$ & - \\
\hline Fig! & $72.7 \mathrm{~s} / \boldsymbol{\nu}$ & $82.2 \mathrm{~s} / \boldsymbol{\nu}$ & $81.8 \mathrm{~s} / \boldsymbol{\nu}^{*}$ & $57.4 \mathrm{~s} / \boldsymbol{\nu}$ & $468.1 \mathrm{~s} / \boldsymbol{V}$ & - \\
\hline Fig 1/as & $359.3 \mathrm{~s} / \boldsymbol{V}$ & $49.2 \mathrm{~s} / \boldsymbol{V}$ & $47.2 \mathrm{~s} / \boldsymbol{\nu}^{*}$ & $29.0 \mathrm{~s} / \boldsymbol{\sim}$ & $757.7 \mathrm{~s} / \boldsymbol{V}$ & - \\
\hline Fig1/nd & $56.9 \mathrm{~s} / \boldsymbol{\sim}$ & $48.7 \mathrm{~s} / \boldsymbol{\sim}$ & $134.2 \mathrm{~s} / \boldsymbol{x}$ & $128.4 \mathrm{~s} / \boldsymbol{x}$ & - & - \\
\hline [9]/2 & $31.0 \mathrm{~s} / \boldsymbol{V}$ & $44.5 \mathrm{~s} / \boldsymbol{V}$ & $96.3 \mathrm{~s} / \boldsymbol{V}^{*}$ & $37.5 \mathrm{~s} / \boldsymbol{V}$ & $258.1 \mathrm{~s} / \boldsymbol{V}$ & - \\
\hline [9]/3 & $43.6 \mathrm{~s} / \boldsymbol{V}$ & $121.6 \mathrm{~s} / \boldsymbol{V}$ & $60.7 \mathrm{~s} / \boldsymbol{\nu}^{*}$ & $87.5 \mathrm{~s} / \boldsymbol{\sim}$ & - & - \\
\hline
\end{tabular}

pre-chart is always an observer and the tableau is used for assumptions. The implementation is taken from [5] and [15]. To isolate the effects under discussion, all experiments use a model that first solves a puzzle to provide some complexity and then adds one or two paths relevant for the LSC.

All experiments use a model which first solves a puzzle to provide some complexity and then adds one or two paths relevant for the LSC.

The first (singleton) group is a time bounded version of the LSC from Fig. 1 on a model not satisfying it due to a condition violation. From the table we can see that the reachability-based approach is significantly faster than the others.

The second group uses a model satisfying the LSC. Its first row is the LSC from Fig. 1, 'as' is a variant with an assumption, and 'nd' is a non-determistic variant where the condition Operational is moved downwards such that it is no longer bonded. In this group, the table indicates that the reachability approach is not always faster, in particular if the property actually holds. In case 'nd' we remain inconclusive whether the system satisfies the LSC or not. The ' $\boldsymbol{V}$ ' results of the reachability way only indicate that there is no safety violation. The following two ' $\boldsymbol{X}$ ' results only indicate that it's possible to avoid a fair state.

The last group is the example from [9], an untimed but highly concurrent LSC with extraordinary large corresponding formulae. It uses only 6 (9) messages to require that 2 (3) agents are started concurrently, then work concurrently, and then report back concurrently. This LSC is bonded, but not time bounded. From the table we can see that the observer approach allows to establish the property in the given amount of time and space while the formula doesn't.

Noticeable in the table is that the LTL way is always terminated prematurely, even if the preceding puzzle is removed. If the model has only a single trace and the LSC is changed s.t. it comprises no timing requirements and no concurrency, the task completes within $\sim 1$ h, thus the LTL way seems rather impractical.

\section{Conclusion}

Although LSC model-checking is basically solved as each LSC has an equivalent temporal logic formula, in practice direct model-checking of the formula is prohibitively expensive due to its size. Our results indicate that full model-checking 
power is only necessary for non-bonded LSCs which occur seldom in practice. In contrast, bonded, time-bounded LSCs are as easy as a reachability problem. To the practically most relevant class of bonded, non-time-bounded LSCs, an approach in between both techniques applies that uses an observer automaton and a small fixed liveness formula. Experimental data indicates that it's beneficial to apply the reachability approach even to non-time-bounded LSCs as contradictions are found faster.

Although our criteria classify most practically occurring LSCs correctly, it is of academic interest to have an algorithm deciding time boundedness. It would be a minor improvement to identify more states as deterministic by more sophisticated procedures.

\section{References}

1. Damm, W., Harel, D.: LSCs: Breathing Life into Message Sequence Charts. Formal Methods in System Design 19 (2001) 45-80

2. Westphal, B., Toben, T.: The good, the bad and the ugly: Well-formedness of Live Sequence Charts. In Baresi, L., Heckel, R., eds.: Proc. FASE 2006 . Volume 3922 of LNCS. (2006) 230-246

3. Toben, T., Westphal, B.: On the expressive power of LSCs. In Wiedermann, J., et al., eds.: Proc. SOFSEM 2006. Volume 2., Inst. of CS, AS, Prague (2006) 33-43

4. Thomas, W.: Automata on infinite objects. In van Leeuwen, J., ed.: Handbook of theor. comp. sc. (vol. B): Formal Models And Semantics. MIT (1990) 133-191

5. Klose, J.: Live Sequence Charts: A Graphical Formalism for the Specification of Communication Behavior. PhD thesis, C. v. O. Universität Oldenburg (2003)

6. Bohn, J., Damm, W., Wittke, H., Klose, J., Moik, A.: Modelling and validating train system applications using statemate and live sequence charts. In: Proc. IDPT 2002, Society for Design and Process Science (2002)

7. Schinz, I., Toben, T., Mrugalla, C., Westphal, B.: The Rhapsody UML Verification Environment. In Cuellar, J.R., Liu, Z., eds.: Proc. SEFM 2004. (2004) 174-183

8. Westphal, B.: LSC verification for UML models with unbounded creation and destruction. In Byron Cook, Scott Stoller, W.V., ed.: Proc. SoftMC 2005. Volume 144:3 of ENTCS., Elsevier B.V. (2005) 133-145

9. Toben, T., Westphal, B.: Concurrent LSC verification. In Lazic, R., ed.: Proc. AVoCS 2005. Volume 145 of ENTCS., Elsevier B. V. (2006) 95-111

10. Bunker, A., Gopalakrishnan, G., Slink, K.: Live sequence charts applied to hardware requirements specification and verification: A VCI bus interface model. Software Tools for Technology Transfer 7 (2004) 341-350

11. Harel, D., Kugler, H., Marelly, R., Pnueli, A.: Smart play-out of behvioral requirements. In: Proc. FMCAD'02. (2002) 378-398

12. Harel, D., Marelly, R.: Come, Let's Play. Springer (2003)

13. Sun, J., Dong, J.S.: Model checking Live Sequence Charts. In: ICECCS, IEEE Computer Society (2005) 529-538

14. Grégoire, B.: Automata oriented program verification. Master's thesis, Facultés Universitaires Notre-Dame de la Paix, Namur (2002)

15. Wittke, H.: A Framework for Specification Verification for Complex Embedded Systems. PhD thesis, C. v. O. Universität Oldenburg (2005) 
16. Schlör, R.C.: Symbolic Timing Diagrams: A Visual Formalism for Model Verification. PhD thesis, C.v. O. Universität Oldenburg (2000)

17. Kugler, H., et al.: Temporal logic for scenario-based specifications. In Halbwachs, N., Zuck, L.D., eds.: Proc. TACAS 2005. Volume 3440 of LNCS. (2005)

18. Brill, M., et al.: Formal verification of LSCs in the development process. In Ehrig, H., et al., eds.: SoftSpez. Number 3147 in LNCS. Springer-Verlag (2004) 494-516 